\title{
resumen sobre tecnología de suelos
}

«Journal of the PCA Research and Development Laboratories», vol. 4, núm. 1, enero 1962.

\section{resumen}

Recoge las experiencias internacionales de laboratorio y trabajos de campo, durante más de un cuarto de siglo, sobre mezclas controladas científicamente de suelo-cemento, y revisa los conocimientos y principios científicos y tecnológicos propios del tema. Para obtener un material estructural único como el suelo-cemento, puede ser necesario emplear técnicas distintas e independientes. Su empleo incluye los pavimentos baratos para carreteras y caminos, la base y subbase para pistas de aeropuertos y carreteras, las medidas contra la erosión en malecones, diques y terraplenes, los lechos de canales y otras muchas aplicaciones de ingeniería, etc. Este resumen se limita solamente a la aplicación del cemento a suelos de grano fino, principalmente, con el fin de obtener un material estructural barato de suelo-cemento compactado para pavimentos.

Las investigaciones, ensayos y experiencias han demostrado que el éxito de los pavimentos de suelo-cemento compactado está gobernado, principalmente, por tres factores de control básicos:

1. Humedad.

2. Densidad.

3. Cemento.

Cada uno de estos factores contribuye, de una manera fundamental, a los resultados obtenidos. Por otra parte, el entendimiento de las relaciones mutuas entre el cemento portland y el suelo dependen de otros tres factores principales:

1. Las propiedades físico-químicas de los suelos.

2. La química del cemento portland.

3. Las propiedades físico-químicas de las mezclas del suelo-cemento antes, durante y después de la hidratación del cemento.

Se discuten los factores exteriores con el fin de valorar la tecnología del suelo-cemento en ensayos e investigaciones de laboratorio, en la proporción básica de cemento, suelo y agua para obtener un material estructural, y en el control de campo y construcción del suelo-cemento compactado. 


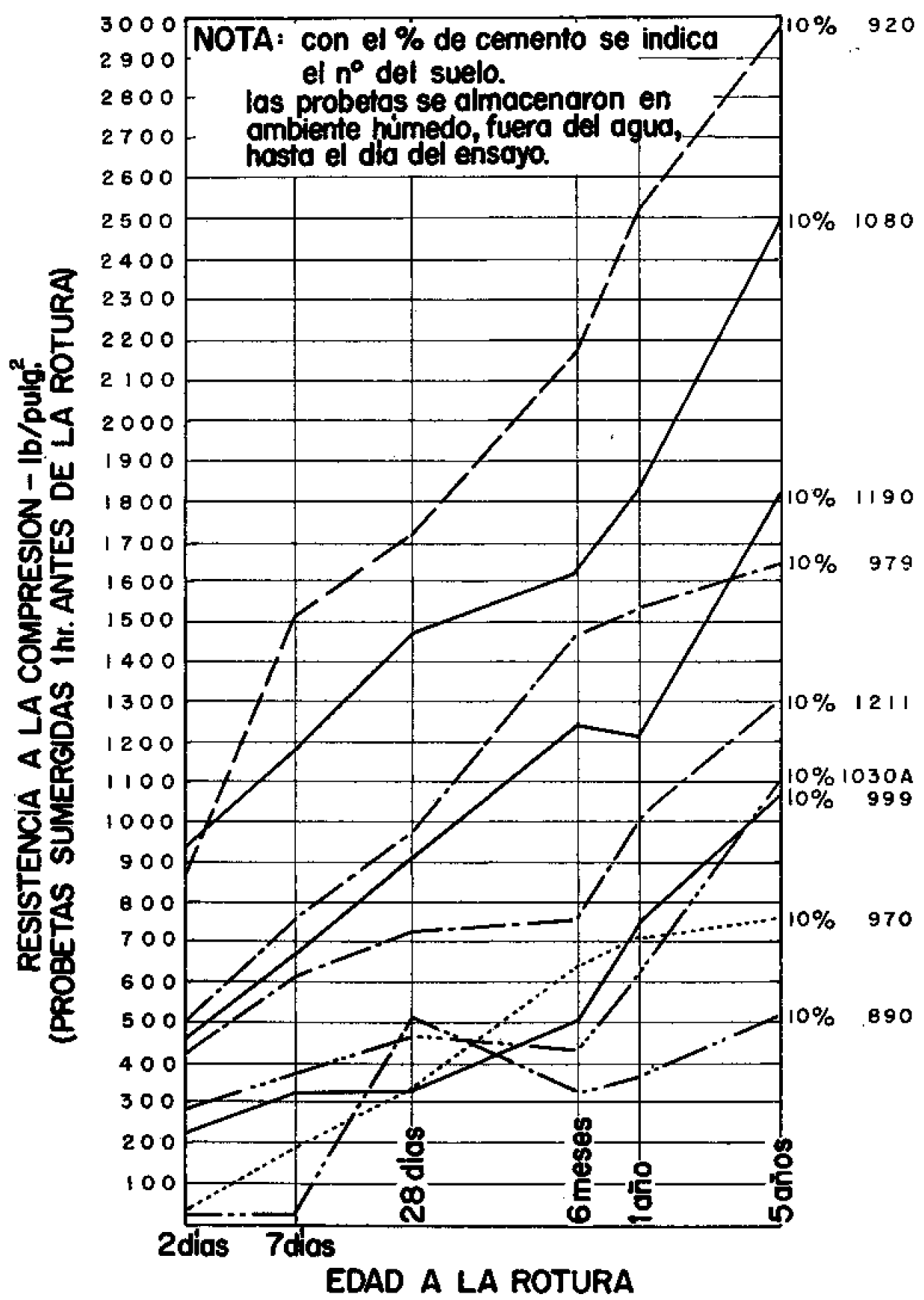

Fig. 2. La resistencin a la compresion del autocomento eompactedo aumenta con la edad. 


\section{Cemento Portland: ASTM Tipo I" .}

Contenido de cemento: Tanto por ciento sobre volumen aparente de mezcla compactada ${ }^{*}$.

Criterios para determinar el cemento requerido por el suelo-cemento compactado para pavimentos: Basado en los ensayos de humedad-sequedad y hielo-deshielo ASTM. Designaciones $\mathrm{D} 599$ y $\mathrm{D} 560$ (ver * página anterior).

1. Las pérdidasas del suelo-cemento durante 12 ciclos de cualquiera de los dos ensayos de sequedad-humedad o hielo-deshielo, deberán cumplir los siguientes límites:

Los suelos de los grupos * A-1, A-2-4, A-2-5 y A-3, no más del $14 \%$.

Los suelos de los grupos A-2-6, A-2-7, A-4 y A-5, no más del $10 \%$.

Los suelos de los grupos A-6 y A-7, no más del $7 \%$.

2. El volumen máximo en cualquier momento durante los ensayos de humedad-sequedad o hielo-deshielo, no excederá en más del $2 \%$ del volumen en el momento de moldeo.

3. El máximo contenido de humedad en cualquier momento mientras duran los ensayos de humedad-sequedad o hielo-deshielo, no deberá exceder de la cantidad con la cual se han lienado totalmente los huecos de la probeta en el momento del moldeo ${ }^{* \$ a}$.

4. La resistencia por compresión de las probetas de suelo-cemento, sumergidas en agua de 1 a 4 horas antes del ensayo, deberá aumentar con la edad y con el contenido de cemento, y sobre este contenido de cemento han de observarse siempre los requerimientos 1,2 y 3 .

Suelo-cemento plástico $(6,7)$ : Mezclas de suelo-cemento con consistencia de mortero. Se emplean para revestimientos de zanjas, acequias, etc.. conde no puedan emplearse los métodos de construcción con suelo-cemento compactado.

Suelo-cemento modificado $(6,8,9)$ : Mezclas de suelo-cemento que contienen cemento sufciente para reducir el contenido de arcilla y coloides (por aglomeración con el cemento) y cumplir las normas aplicables a suelos en cuanto a granulometría, contenido de arcilla, límites de Atterberg, etc.

Bases tratadas con cemento $(10,11)$ : Mezclas de suelo-cemento compuestas generaimente for materiales granulares que se salen de la norma, que se mejoran con adición de cemento para obtener un material que cumpla las especificaciones aplicables a sub-bases para pavimentos de hormigón.

Bases para analizar la influencia del cemento: $\mathrm{El}$ contenido de cemento de las muestras de ensayo de mezclas de suelo-cemento se basa sobre el tanto por ciento en volumen con el fin de que puedan prepararse muestras de ensayo de muy diferentes pesos secos por pie cúbico, muy

* Otros tipos producirán los mismos resultados prácticos.

* Un pie cúbico del cemento considerado pesa 94 libras; asi, un ple cúbico de suelo compactado con un contenido del $10 \%$ de cemento en volumen contiene 9,4 libras de cemento.

** Grupo de suelo AASHO.

*** La experiencia ha demostrado que este criterio sobre cantidad de cemento requerido no es general para todos los suelos que se encuentran en U. S. Era el de más valor durante las investigaciones iniciales, ya que comprobó los conceptos de resistencias obtenidos en el ensayo. Su uso se ha limitado a los suelos muy expansivos, poco corrientes, y para proyectos de investigación más bien que con objeto de determinar e] cemento requerfdo para proyectos de construcción. 
diferentes contenidos de huecos y de muy diferente composición físico-química, con la misma cantidad (mismo número de granos) de cemento en cada muestra. Esto permite una comparación sencilla y directa de la influencia del cemento sobre suelos de muy diversas características. Todos los puntos de esta publicación se basan en este principio.

Proyecto del pavimento $(11,12,13,14,15,16)$ : Una parte del tema no incluido en esta publicación. Primero se determina la mínima cantidad de cemento para obtener un material estructural, por los procedimientos discutidos en este trabajo; a continuación se determinan las propiedades estructurales (tales como las resistencias por composición y flexión) para cada mezcla de suelo y cemento, puesto que ellas varían con cada suelo específico $(15,16)$. Estas propiedades estructurales, junto con las características de sub-base, clima y tráfico, deben emplearse para el proyecto de los pavimentos. Si fuesen necesarias mayores resistencias pueden obtenerse aumentando el contenido de cemento sobre el mínimo requerido para producir un material estructural.

Construcción del pavimento $(3,17)$ : (No incluida en esta publicación).

\section{consideraciones previas y control}

Teóricamente, los suelos se consideran como una masa desmenuzada, compuesta por sus correspondientes tamaños de grano del suelo que forman el árido de las mezclas de suelo-cemento.

Las relaciones del contenido de humedad del suelo desmenuzado, la energía de compactación con las densidades resultantes y las resistencias mecánicas del suelo compactado fueron establecidas hace treinta años en la construcción de presas de tierra (18). Se demostró que empleando una energía de compactación " previamente determinada en el laboratorio, se alcanza una densidad máxima con un contenido de humedad que se considera óptimo. También se demostró que este óptimo de humedad y máxima densidad producía un suelo compactado que tenía un máximo de resistencia para la energía de compactación empleada.

Las primeras investigaciones científicas $(1,5,19,20)$ sobre mezclas de suelo-cemento demostraron que estos mismos principios y relaciones eran válidos para mezclas de suelo pulverizado y cemento, cuando eran compactadas inmediatamente después de mezcladas y antes que la hidratación del cemento tuviera lugar.

Este primer paso para confirmar la validez de las relaciones humedad-densidad con suelo pulverizado que contiene cemento, suministró también datos para contestar a la pregunta clave: ¿El agua libre presente en la humedad óptima, será la adecuada para la hidratación del cemento contenido en la mezcla? La respuesta fue: «Sís».

Este concepto fundamental fue válidó para el desarrollo de esta investigación inicial. Entonces se sentó la hipótesis de que puesto que el suelo que se compactó con su humedad óptima a máxima densidad produjo la máxima resistencia (y soportaría el apisonado con el rodillo de pata de cabra), adquiriría la resistencia adecuada para pavimentos de bajo costo. Sin embargo, posteriores aumentos en el contenido de humedad rebajarían esta máxima resistencia, a menos que el cemento incorporado antes de la compactación hidratara subsiguientemente, de tal manera, que mantuviera la masa compacta a la máxima densidad independientemente de los cambios posteriores en el contenido de humedad.

* Determinada por ensayo para simular una compactación en el terreno con rodtllo de pata de cabra. 
Esta hipótesis condujo al desarrollo de los ensayos de humedad-sequedad y hielo-deshielo para valorar la reacción del suelo con el cemento y determinar las cantidades de conglomerante requeridas para mantener la masa unida produciendo un material estructural, puesto que se encontró que la adición de cemento perturbaba los conceptos fundamentales que llevan consigo otros procedimientos de ensayo posibles, comunes a suelos y hormigones.

Sin embargo, se dio una consideración especial a varios ensayos de compresión y tracción aceptados para el hormigón que podían ser modificados para simular las fuerzas internas de expansión y contracción (retracción) producidas por cambios de humedad en las masas de suelo de las que constituye la mayor parte (aproximadamente, $85-90 \%$ ) de la mezcla. No obstante, la comparación de estas fuerzas aclaró que no eran aplicables. Por otra parte, las alternancias - ciclos de humedad-sequedad y hielo-deshielo provocarían unas fuerzas internas similares y comparables a las producidas por los cambios del contenido de bumedad. Se encontró que doce ciclos de ensayo eran los adecuados y fueron adoptados para uso en el laboratorio.

El ensayo de humedad-sequedad se fijó en principio para simular las fuerzas de contracción (retracción). Se reconoció que las temperaturas en la construcción estarían por debajo de la temperatura máxima de ensayo y que esta máxima temperatura aceleraría la hidratación del cemento.

El ensayo de hielo-deshielo se fijó en principio para simular las fuerzas internas de expansión producidas por la humedad en los stuelos de grano más finó. También evita la hidratación acelerada del cemento presente en el ensayo de humedad-sequedad, puesto que las temperaturas ambiente son las que gobiernan la máxima temperatura.

Ambos ensayos, adaptados ya por las normas ASTM, AASHO y ASA, usados con el correspondiente criterio para determinar el mínimo contenido de cemento necesario para obtener un material estructural apto para la construcción de suelo-cemento compactado, son la base para el ensayo, construcción y uso con garantías y éxito. También se pueden emplear otros métodos, relacionados con los ensayos de humedad-sequedad y hielo-deshielo $(21,22,23,24)$ para determinar los contenidos de cemento en la construcción de suelo-cemento compactado. Los veintiséis años de puesta en servicio en todo el territorio de los EE. UU. demuestran que los contenidos de cemento asi determinados son los adecuados para resistir a las amplias diferencias de exposición a la intemperie. También, estos proyectos puestos en servicio han demostrado que las reacciones químicas y fisicas son estables a largo plazo, es decir, no son reversibles. Igualmente importante es el hecho de que el suelo-cemento compactado contiene huecos de varios tamaños, que oscilan del 30 al $40 \%(5,20)$, lo cual se relaciona con los principios de durabilidad que lleva consigo el aire ocluido en la técnica del hormigón.

En este momento será útil discutir e ilustrar qué ocurre en una mezcla de suelo y cemento cuando se aumenta progresivamente la cantidad de cemento. El primer punto importante y básico es que las partículas de cemento están rodeadas por los granos de suelo y que la mezcla está hímeda, no mojada, en su contenido de humedad optimo. Apretando fuertemente un puñado de mezcla de suelo-cemento, que contenga el óptimo de humedad, en su mano, dejará ésta solamente húmeda. Por comparación, en el hormigón, los áridos finos y gruesos están envueltos por el cemento y con el agua suficiente para producir una mezcla plástica.

El recubrimiento de las partículas de cemento por el suelo se muestra en la figura 1. En el esquema de la izquierda «A», solamente se agregó una pequeña cantidad de cemento $(2 \%)$ a la mezcla compactada. Bajo el esfuerzo aplicado para obtener la máxima compactación, los granos de suelo que la rodean son forzados a entrar en contacto íntimo con la partícula de cemento. Al hidratarse el cemento, el producto resultante es una aglomeración de granos de suelo-cemento entremezclados en la masa de suelo. Los tamaños de los granos de la masa resultante aumentan, y la capacidad de retención de agua disminuye. Este fenómeno, cuando se emplean pequeñas cantidades de cemento, produce un stelo corregido con cemento.

En el esquema de la derecha «B» (fig. 1) se agregó suficiente cemento ( $10 \%$ ) para desarrollar cadenas continuas y eslabones o anillos de cemento y granos de suelo, con objeto de producir suelo-cemento compactado. 


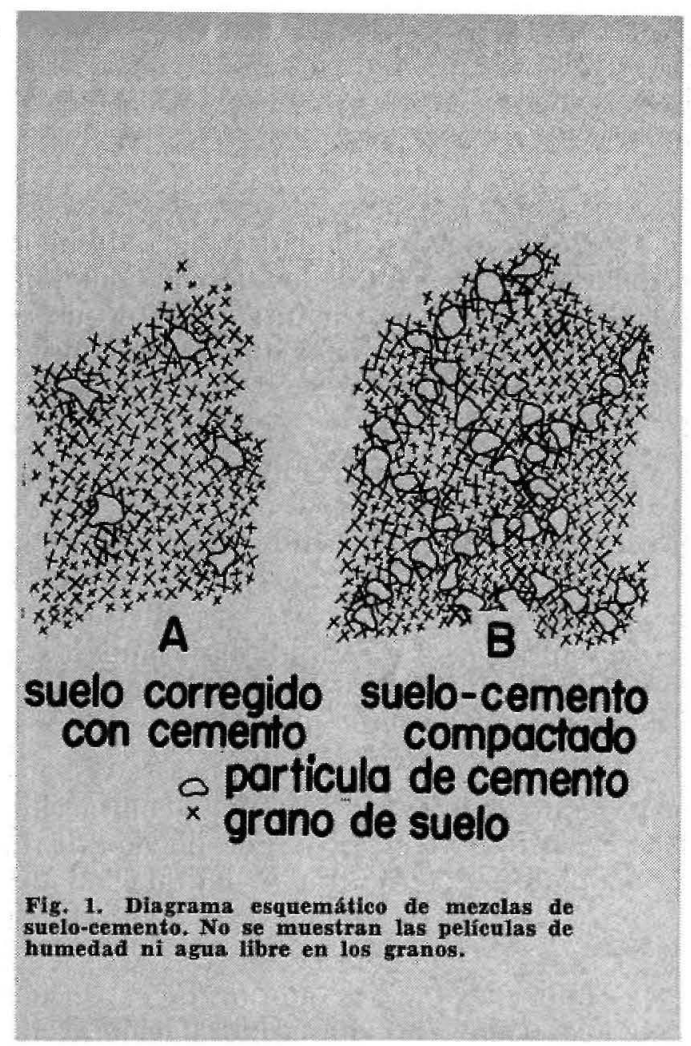

Hay bolsas rellenas únicamente por el material del suelo, que se mantienen fijas por los granos de cemento hidratado que le rodean y mantienen adheridos los granos de suelo.

El esquema «B» representa solamente la fracción de suelo-cemento, compuesta por cemento $\mathrm{y}$ el material que pasa por el tamiz núm. 10. La cantidad de esta fracción cementada (material semejante a un mortero) debe ser suficiente para encerrar ampliamente todo el material de mayor tamaño en la mezcla.

\section{La influencia del suelo}

Los contenidos de cemento requeridos por los suelo-cementos compactados oscilan del 6 al $16 \%$ en volumen del volumen compactado. Las investigaciones, ensayos y experiencias han desarrollado una información que demuestra que hay muchos factores del suèlo que afectan las relaciones suelo-cemento. Ninguno de estos factores ni un grupo reconocido de ellos, tiene una influencia predominante y previsible sobre las reacciones entre el suelo y el cemento. Conocidas las generalidades sobre estos factores se encontrarán con alguna frecuencia, ciertas anomalías y reveses en la aplicación.

\section{El tamaño del grano}

En general, a medida que aumenta en una mezcla la cantidad de limo, arcilla y coloides, la cantidad de cemento requerida para conseguir el suelo-cemento compactado es mayor. El material que pasa por el tamiz del núm. 10 debe ser suficiente, con su contenido de cemento, para llenar los huecos del material grueso que retiene el tamiz núm. 10. De este modo, la mezcla del material que pasa por el tamiz núm. $10 \mathrm{y}$ el cemento viene a ser como un mortero húmedo que rodea completamente a la fracción de gruesos de la mezcla y controla predominantemente las características de la misma.

A medida que el tamaño de grano del suelo decrece, la humedad óptima aumenta, la densidad máxima disminuye y la cantidad de cemento necesario para un suelo-cemento compactado aumenta. Tomando como referencia las humedades óptimas se saca, como conclusión general, que los suelos con el contenido óptimo de humedad más alto requieren el mayor contenido de cemento. Del mismo modo pueden sacarse conclusiones generales cualitativamente (20) comparando las densidades, los índices de plasticidad, los límites de retracción y otros límites de Atterberg.

\section{Propiedades del suelo}

Las propiedades intrínsecas de los granos del suelo varían ampliamente, y parece que tienen una mayor influencia sobre las reacciones entre el suelo y el cemento que todos los demás factores y que muchos de ellos están concretamente identificados y valorados. Sin embargo, se ha puesto en claro la evidencia de su importancia y existencia. Suelos de superficie arenosa en regiones con vegetación de coníferas, poseen algún atributo físico-químico que inhibe la hidratación normal del cemento. Cuando se comparan con otros suelos de granulometría y límites 
de Atterberg similares, o con suelos de horizonte más bajo en la misma zona, requieren mayores cantidades de cemento para obtener el suelo-cemento compactado. Este factor físico-químico, particulamente pernicioso, puede salvarse mediante la adición de pequeñas cantidades de cloruro cálcico, lo que indica una posible reacción química. También puede evitarse mezclando el suelo con un $20 \%$ de arcilla, lo que indica posiblemente tanto una reacción física como química.

Este elemento físico-químico será identificado como de naturaleza orgánica, pero no hay una evidencia clara de que sea fundamentalmente un producto orgánico *. Se considera ser, principalmente, una función de la química de superficie de los suelos que incluye el intercambio iónico, la estructura reticular de los minerales de la arcilla presentes y, posiblemente, la influencia puzolánica.

Hay, sin embargo, mucha evidencia $(5,27)$ de que el ensayo colorimétrico (28) no permite identificar directamente los productos responsables del retraso en la reacción del cemento. Por ejemplo, un estudio extenso (20) ha demostrado que el contenido orgánico de un considerable número de suelos de los grupos A-2 y A-3 que requieren el $8 \%$ de cemento para suelo-cemento compactado tíenen contenidos orgánicos que oscilan de 100 a 30.000 p. p. m. Además, se han desarrollado otros grupos de conocimientos referentes al complejo orgánico y química superficial de los suelos $(29,30,31,32)$ que trazan el camino para nuevos trabajos adicionales que resultarán de mucho interés.

Para resumir la influencia del contenido de materia orgánica (determinación colorimétrica) puede decirse que, a medida que aumenta su contenido, la cantidad de cemento necesario puede aumentar. Algunos suelos con bajo contenido en materia orgánica, han requerido un contenido anormalmente alto de cemento comparados con suelos afectados de factores físicos similares; esto demuestra la influencia de la química superficial del suelo.

\section{Mecánica del suelo}

El Departamento de Agricultura de EE. UU. ha desarrollado un sistema de identificación del suelo (33) basado en la premisa de que suelos de superficie formados bajo el mismo proceso de formación poseen las mismas propiedades físico-químicas. Las investigaciones ìniciales de las mezclas de suelo-cemento $(5,20)$ han demostrado que las características físicas de aquéllas no se corresponden o explican las diversas reacciones de las mezclas suelo-cemento. Se demostró que ciertas características iónicas del suelo eran fundamentalmente importantes (34). También se demostró que suelos idénticos (identificados por este sistema) tenían idénticas reacciones suelo-cemento, esto es, requerían la misma cantidad de cemento para el suelo-cemento compactado $(21,23)$. Todos estos datos demostraron más tarde que estas propiedades físicas o químicas directas de los suelos, tales como granulometría, índice de plasticidad, límite de retracción, contenido orgánico, concentración de ton hidrógeno $(\mathrm{pH})$, porcentaje de sólidos a la máxima densidad y otras propiedades similares, no se corresponden, aisladamente o en grupos, con las exigencias de cemento. Por ejemplo, demostrando una vez más la influencia de la química de superficie, los suelos rojos y amarillos subtropicales del sureste de los EE. UU. de un modo general requieren menos cemento que los suelos de similar granulometría del centro norte de EE. UU.

\section{Resumen de las influencies del suelo}

Los actuales métodos que valoran las propiedades físico-químicas de los suelos no revelan las relaciones fundamentales de las mezclas de suelo-cemento. Sin embargo, los suelos identificados por el sistema geotécnico empleado en los EE. UU., como suelos idénticos, poseen propiedades físico-químicas idénticas con idénticas relaciones suelo-cemento. También las exploraciones en el campo de la química de superficie de los suelos, estructura reticular de los mine-

* Pequeñas cantidades de carbón, lignito, raices y materiales orgánicos símilares no son perniciosos para la hidrataclón del cemento. 


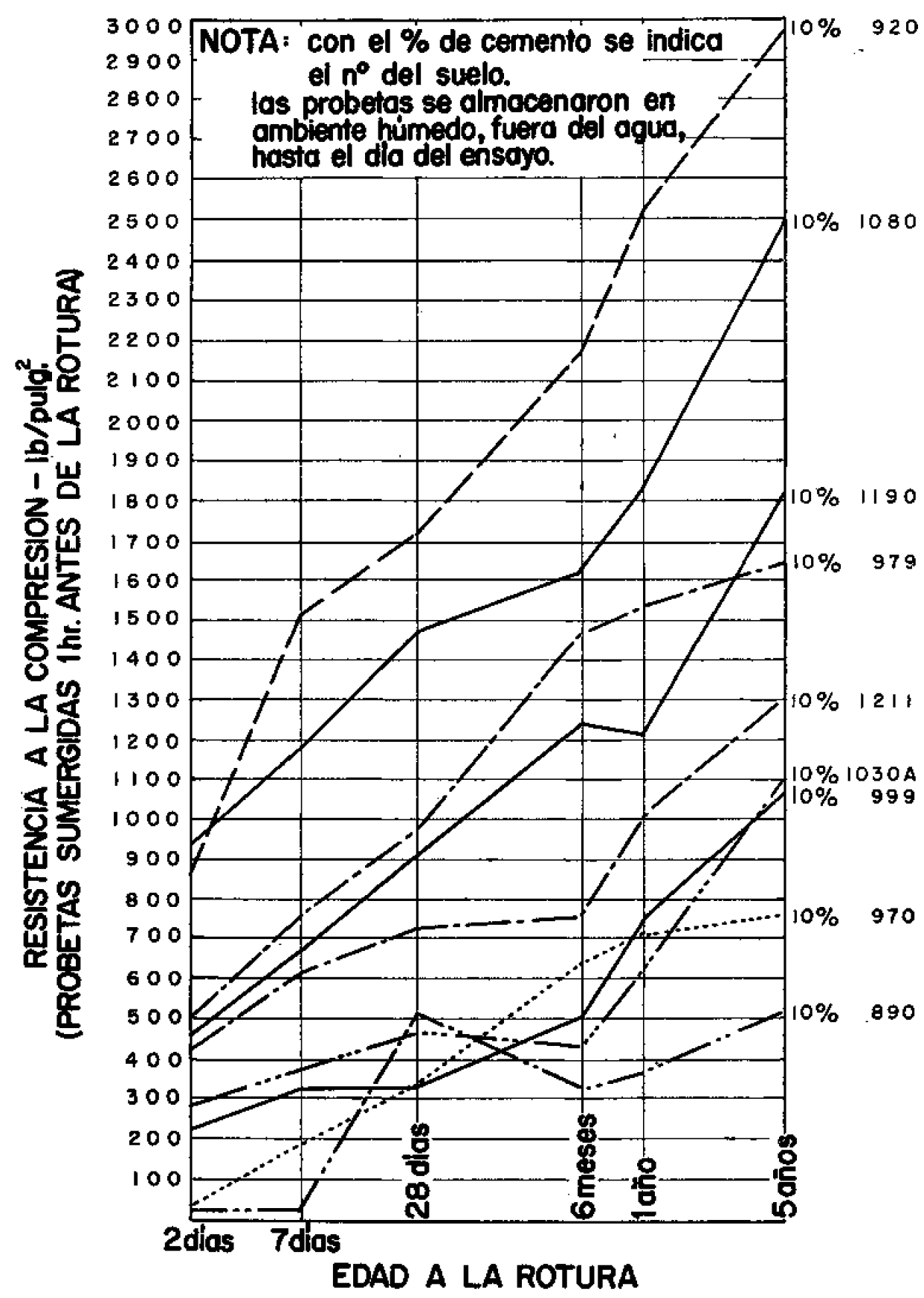

Fig. 2. La resistencia a la compresión del suelocemento compactado anmenta con la odad. 
rales arcillosos y composición química de los suelos prometen proporcionar una valiosa información sobre las reacciones del suelo y cemento.

\section{Influencia del cemento}

Las referencias citadas anteriormente suministran datos que demuestran que la acción del cemento en las mezclas de suelo-cemento sigue los mismos principios básicos que en el hormigón, siendo la diferencia fundamental, como antes se dijo, que en los suelos-cementos compactados las partículas de cemento resultan estar envueltas por los granos de suelo. También este dato demuestra claramente que la composición y características del cemento desempeñan un papel fundamental, de la mayor importancia en la tecnología del suelo-cemento. Además, la velocidad y eficacia de la hidratación del cemento y la velocidad en el aumento de resistencias, están directamente relacionadas con la cantidad de cemento agregado y el suelo particular empleado $(5,19,20,35)$. Los datos suministrados por la figura 2 y la tabla 1 demuestran que la resistencia por compresión del suelo-cemento compactado aumenta con la edad (35).

TABLA 1.-Resultados de ensayos de suelos.

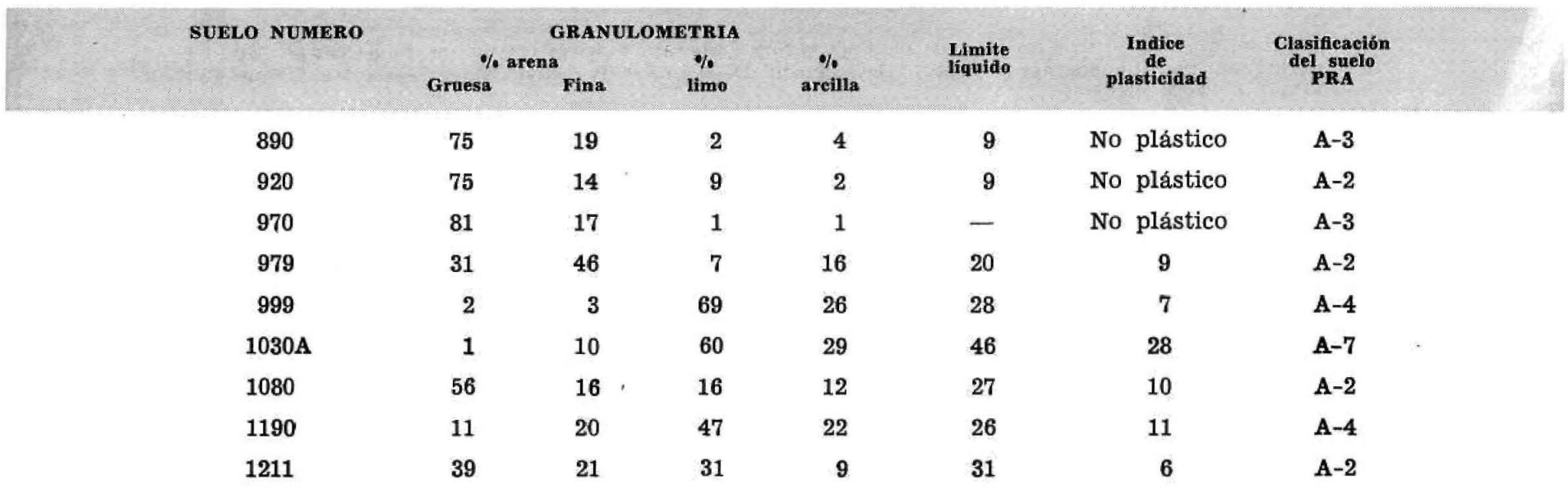

La valoración de la influencia del cemento incluye diversos factores directamente relacionados con la construcción $(5,6,20,36)$. Las velocidades de hidratación del cemento y el tiempo de ejecución son particularmente importantes y se consideran en las referencias citadas. También se incluyen en estas referencias las propiedades del cemento y el fenómeno de hidratación del cemento en relación con las mezclas de suelo-cemento.

La interacción química de las mezclas de suelo-cemento es demostrada por una serie de investigaciones $(34,37,38,39,40,41,42,43,44)$ sobre la influencia de la química superficial de los suelos y de los aditivos químicos al suelo-cemento. Esto se evidencia, generalmente, por los resultados físicos finales. Continuadas investigaciones en este campo, así como en el que relaciona la estructura reticular y las posibles influencias puzolánicas, definirán más claramente las reacciones fundamentales de suelo y cemento.

En resumen: las propiedades del cemento y su proceso de hidratación en el suelo-cemento compactado son, en general, las mismas que en el hormigón, con las modificaciones propias producidas por las características físico-químicas combinadas del suelo $\mathrm{y}$ del cemento. 


\section{Conclusión}

Las relaciones suelo-cemento son un complejo de propiedades físico-químicas de los snelos. de la química del cemento y de las propiedades físico-químicas de la mezcla suelo-cemento. Están en marcha en la PCA (Research and Development I_aboratories), investigaciones sobre los factores básicos que gobiernan estas propiedades.

Se ha demostrado durante un cuarto de siglo de experiencias sobre suelo-cemento aplicado a carreteras que los ensayos de humedad-sequedad y de hielo-deshielo, aplicados con criterio adecuado para determinar el mínimo contenido de cemento para producir un material estructural para suelo-cemento compactado, han revalorizado felizmente estos factores básicos de tipo empírico. Por otra parte, se ha demostrado también que la interacción química de las mezclas suelo-cemento es estable por largos períodos de tiempo, es decir, que no es reversible. El uso en la construcción de este contenido de cemento previamente fijado, la apropiada humedad óptima, la densidad máxima de la mezcla, y otras especificaciones aplicables en cada caso, han producido resultados satisfactorios en una gran variedad de suelos y amplia gama de condiciones geográficas y meteorológicas.

\section{bibliografia}

(1) Catton, Miles D.: "Progress Report on Exploratory Laboratory Investigation of Boll-Cement Mixtures". An unpublished mimeographed report, Development Department, Portland Cement Association, Chicago, Illinois (May 1, 1936), 106 pages.

(2) Sheets, F. 'T., and Catton, Miles D.: "Basic Princlples of Soil-Cement Mixtures", Engineering NewsRecord, 120, 869-875 (June 23, 1938).

(3) Sheets, F. T., and Catton, Miles D.: "How to Process Soil-Cement Roads", Engineering News-Record, $121,20-24$ (July 7,1938 ).

(4) Editorial: "Science Brought to Aid", Engineering News-Record, 121, page 10 (July 7, 1938).

(5) Catton, Miles D.: "Basic Principles of Soil-Cement Mixtures and Exploratory Laboratory Results", Highway Research Boarl Proceedings, Part II (A Symposium on Soil-Cement Mixtures for Roads), 17, 7-31 (1937).

(6) Catton, Miles D.: "Goil-Cement: A Construction Material", reprinted from Proceedings of Conference on Soil Stabilization, at Massachusetts Institute of Technology (June 18, 19, 20, 1952), 18 pages.

(7) - Byrne, W. S. and Holtz, W. G.: "Soil-Cement Lining Placed Mechanically", Engineering News-Record, 139, 860-863 (December 25, 1947).

(8) Catton, Miles D.: "Laboratory Investigations of Soil-Cement Mixtures for Subgrade Treatment in Kansas", Higway Research Board Proceedings, Part II (A Sympasium on Soil-Cement Mixtures for Roads), $17,92-96(1937)$.

(9) Reid, C. R.: "Concrete Pavement Bubgrade, Design, Construction, Control", Highway Researcr Baard Proceedings, 19, 541-551 (1939).

(10) Hveem, F. N.: "Construction Practices on Cement-Treated Subgrades for Concrete Pavements", Hightoay Research Board Bulletin 265, 1-7 (1960).

(11) Abrams, M. S.: "Laboratory and Field Tests of Granular Boil-Cement Mixtures for Base Courses", American Society for Testing Materials STP No. 254, 229-243 (1959). Reprinted as Development Department Bulletin D37, Portland Cement Assoctation,

(12) "Thlekness of Flexible Pavement", Highway Research Board, Curtent Road Ploblems No. 8-R (Rev. Ed.), 49 pages (November, 1949).

(13) Hveem, F. N., and Carmany, R. M.: "The Factors Underlying the Rational Design of Favements", Highway Research Board Proceedings, 28, 101-136 (1948).

(14) "Design of Flexible Pavement Using the Triaxial Compression Test", reported by the State Highway Commission of Kansas, Highway Research Board Bulletin No. 8 (1947), 63 pages.

(15) Felt, E. J., and Abrams, M. S.; "Btrength and Elastic Properties of Compacted Soil-Cement Mixtures", American Sodiety for Testing Materials STP No, 206, 152-178 (1937). Feprinted as Development Department Bulletin D16, Fortland Cement Association.

(16) Balmer, G. G.: "Shear Strength and Elastic Properties of Soll-Cement Mixtures under Triaxial Loading", American Society for Testing Materials Prccedings, 58, 1187-1204 (1958), Reprinted as Development Department Bulletin D32, Portland Cement Association. 
(17) Catton, Miles D.: "Some Wartime Soil-Cement Construction Experiences", Highway Research Board Proceelings, 24, 450-466 (1944). (Also, numerous articles on construction procedures which have been published by Engineering News-Record, New York, N. Y, and by Roads and Streets, Chicago, Illinois.)

(18) Proctor, R. R.: four articles on "Design and Construction of Rolled-Earth Dams" (various titles), 111, Engineering News-Record (1933): August 31, page 245; September 7, page 286; September 21, page 348; and September 28, page 372 .

(19) Catton, Miles D.: "Early Soil-Cement Research and Development" (Paper No. 1899) Journal of the Highway Division, Proceedings of the American Society of Civtl Engineers, 85, HW 1, 1-16 (January, 1959) Discussion, 85, HW 3, 77-93 (September, 1959); Author's Closure, 86, HW 1, 67-76 (March, 1960). Reprinted as Development Deparment Bulletin D42, Portland Cement Association.

(20) Catton, Miles D.: "Research on the Physical Relations of Soil and Soil-Cement Mixtures", Highway Research Board Proceedings, 20, 821-855 (1940).

(21) Hicks, L. D.: "Sampling, Soil Classifleation and Ċement Requirements-N. Carolina", Highway Re. search Board Proceedings, 19, 521-523 (1939).

(22) Leadabrand, J. A., and Norling, L. T.: "Soil-Cement Test-Data Correlation in Determining Cement Factors for Sandy Solls", Highway Research Board Bullettn 69, 29-46 (1953).

(23) Leadabrand, J. A., Norling, L. T., and Hurless, A. C.: "Soil Serles as a Basis for Determining Cement Requirements for Soll-Cement Construction", Highway Research Board Bulletin 148, 17 pages (1957).

(24) Norling, L. T., and Packard, R, G.: "Expanded Short-cut Test Method for Determining Cement Factors for Sandy Soils", Highway Research Board Builetin 197, 20-31 (1958).

(25) Powers, T. C.: "The Air Requirements of Frost Resistant Concrete", Highoay Research Board Proceedings, I.P, 184-202 (1949).

(26) Klieger, Paul: "Effect of Entrained Air on Strength and Durability of Concrete Made which various Maximum Sizes of Aggregate", Highway Research Board Proceedings, 31, 177-201 (1952).

(27) Catton, Miles D, and Felt, E. J.: "Effect of Sil and Calcium Chloride Admixtures on Boil-Cement Mixtures), Highway Research Board Proceedings, 23, 497-529 (1943).

(28) ASTM Standards, Designation: C 40-60, "Test for Organic Impurities in Sands for Concrete", American Society for Testing Materials.

(29) Clare, K. E., and Sherwood, P. T, (Road Research Laboratory, Harmondsworth, England): "Effect of Organic Matter on the Betting of Soil-Cement Mixtures", Journal of Applied Chemistry (London), 4, 11 $625-630(1954)$.

(30) "Location of Mrterial Having an Adverse Effect on the Stabilization of Soil with Portiand Cement" Military Engineering Experimental Establishment (British), Report No. 463 (November, 1953 ).

(31) Sherwood, F. T.: "The Stabllization with Cement of Weathered and Sulphate-Bearing Clays", Geotechnic, VII, 4, 179-191 (December, 1957).

(32) Robbins, ๓. G., and Mueller, P. E.: "Development of a Test for Identifying Poorly Reacting Sandy Soils Fncountered in Soil-Cement Construction", Highway Research Board Bulletin 267, 46-50 .1960).

(33) Kellogg, C. E.: "Development and Significance of the Great Soll Groups of the United States", U. S. Department of Agriculture Miscellaneous Publication No. 229 (1936).

(34) Winterkorn, H. F.; Glbbs, H. J., and Fehrman, R. E.: "Surface Chemlcal Factors of Importance in the Hardening of Soils by Means of Portland Cement", Highway Research Boarl Proceedings, 22, 385. 414 (194)).

(35) Catton, Miles D.: "Tests Show Soil-Cement Improves with Age", Sotl-Cement News. No. 20, page 2 (May, 1946), publeshed by Portland Cerment Associstion, Chicago, Illinois.

(36) Felt, E. J.: "Factors Influencing Physical Propectfes of Soll-Cement Mixtures", Highway Research Board Bulletin 108, 138-162 (1955). Reprinted as Development Department Bulletin D5, Portland Cement Association.

(37) Mehra, S. R.; Chadda, L. R.; Kapur, R. N.: "Role of Detrimental Salts in Soil Stabilization, with and wthout Cement-I. The Effect of Sodium Sulphate", Indian Concrete Joturnal, 29, 336-397 (October, 1955),

(38) Chadda, L. R., and Raj. Hem.: "Role of Detrimental Salts in soil Stabilization, with and without Ce. ment-2. The Effect of Sodium Carbonate", Indian Concrete Joumal, 29, 401-402 (December, 1955$)$.

(39) Uppal, I. S., and Kapur, B. P.: "Role of Detrimental Salts in Soil Stabilization, with and without Cement-3. The Effect of Magnesium Sulphate", Indian Concrete Journal, 31, 228-231 (July, 1957).

(40) Lambe, T. W, and Moh, Z. C.: "Improvement of Strength of SoiI-Cernent with Additives", Highway Research Board Bulletin 183, 38-47 (1958),

(41) Handy, R. L.: "Cementation of Soil Minerals with Portland Cement or Alkalis", Highway Research Board Bulletin 198 (1958).

(42) Lambe, T. W.; Michaels, A, S, y and Moh, Z. C.: "Improvement of Sotl-Cement with Alkali Metal Compounds", Highway Research Board Bulletin 241, 67-108 (1960),

(43) Lambe, T. W.: "Physico-Chemioal Properties of Solls: Role of Soil Technology", Journal of the sotl Mechanios and Foundation Division, Proceedings of the American Society of Civil Engineers, 85, 55-70, 5M 2 (Aprli, 1959).

(44) Carrol, Dorothy: "Ion Exchange in Clays and Other Minerals", Bulletin of the Geological society of America, 70, 6, 749-780 (June, 1859).

(45) Anderson, L.: "Clay Chemistry in Soil Stabilization", Geologisła föreningen Förhandlingar, Stockholm (In English), 82, 2, 274-297 (1960) 